\title{
EDITORIAL
}

\section{Familial idiopathic basal ganglia calcification: rediscovering an old challenge in organic psychiatric disorders}

\author{
João Ricardo Mendes de Oliveira, ${ }^{1,2}$ Matheus Fernandes de Oliveira ${ }^{3}$ \\ ${ }^{1}$ Keizo Asami Laboratory (LIKA), Universidade Federal de Pernambuco (UFPE), Recife, PE, Brazil. ${ }^{2}$ Neuropsychiatric Department, UFPE, \\ Recife, PE, Brazil. ${ }^{3}$ Neurosurgery Residency Program, Hospital do Servidor Público Estadual de São Paulo, São Paulo, SP, Brazil.
}

Idiopathic basal ganglia calcification (IBGC) is a known organic cause of psychiatric disturbances and remains a challenge in modern neuropsychiatry, in terms of both diagnosis and treatment. Surprisingly, such patients are still often misdiagnosed as having schizophrenia, dementia, or Parkinson's disease. ${ }^{1-3}$

Currently, there is an increasing rate of detection of idiopathic calcifications in neuroimaging procedures, especially in the basal ganglia, but also in other brain sites (Figure 1), during the investigation of milder symptoms, some of them with transient symptomatology, and also in the assessment of asymptomatic subjects with positive family history. Other subjects are diagnosed in emergency rooms or even randomly during the investigation of other pathologies, ${ }^{2,3}$ which suggests that this phenotype is more common than previously assumed. Also, a growing number of cases have been systematically reported in several countries, especially in those where neuroimaging equipment and imaging facilities have become more available, due to a lower price per exam. ${ }^{3}$ The literature reports different prevalence rates for IBCG, ranging from $0.3 \%$ up to $12 \%$ in patients undergoing neuroimaging examinations. ${ }^{1,3}$

Basal ganglia calcification is becoming an increasingly cited topic in the literature and is receiving attention from important research groups, caregiver associations, and funding agencies. Recently, in a collaborative analysis involving China, Brazil, Spain, and Switzerland, the first causative gene was identified and different mutations reported at the SLC20A2 gene, located at chromosome 8 and encoding an inorganic phosphate transporter (PiT2). ${ }^{4,5}$ This transmembrane protein, initially identified as a retrovirus receptor, is expressed in several tissues (e.g., bone, vascular smooth muscle cells, parathyroid glands, kidney, intestine, and the brain), and fulfills a housekeeping function in $\mathrm{Pi}$ homeostasis. ${ }^{4}$ Additional screening analysis showed that this gene is responsible for approximately $50 \%$ of familial cases of IBGC. ${ }^{5}$ More recently, two French families with IBGC were found to have mutations in the platelet-derived growth factor

Correspondence: Matheus Fernandes de Oliveira, Department of Neurosurgery, Hospital do Servidor Público Estadual de São Paulo, Rua Pedro de Toledo, 1800, CEP 04029-000, São Paulo, SP, Brazil. E-mail: mafernoliv@yahoo.com.br receptor beta (PDGFRB) gene, opening a new avenue for a better understanding of this phenotype as being caused by different molecular failures in mechanisms of vascular homeostasis. ${ }^{6}$ Considering the two genes so far identified, responsible for what is assumed to be less that $60 \%$ of the all cases of IBGC, we predict that additional new genes will be discovered to clarify the genetic basis of more families. This finding also opens a new therapeutic target based on $\mathrm{Pi}$ homeostasis, perhaps with the potential use of bisphosphonates to modulate calcium accumulation.

Nevertheless, these findings do not mean that we should implement computed tomography (CT) scans as a routine procedure to find every basal ganglia calcification around us. Previous results were not convincing. ${ }^{7}$ Indeed,

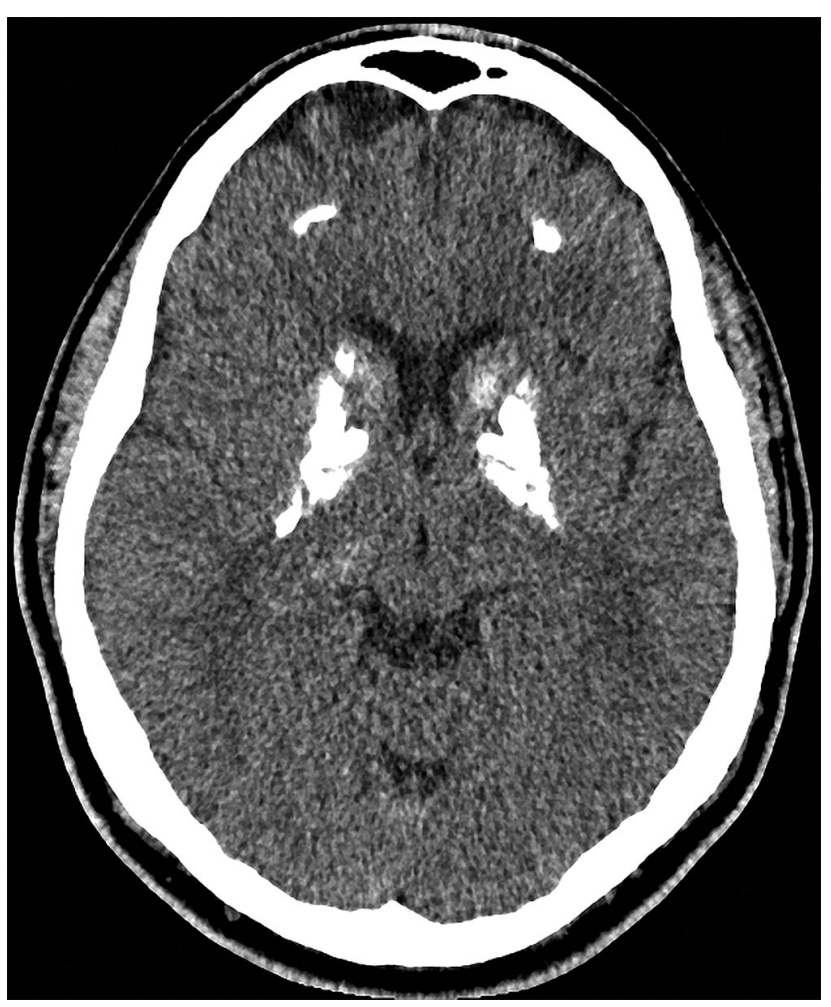

Figure 1 Patterns of brain calcifications often found in patients with idiopathic basal ganglia calcification 
in general, patients with a psychiatric profile will only undergo neuroimaging investigation if the most prevalent diagnoses are excluded, when response to treatment is too poor, or when an unusual course of disease, with rapid deterioration, is observed. Over a 3-year period, McClellan et al. screened all inpatients at a psychiatry unit with a routine CT exam, and only two cases out of 261 patients examined showed basal ganglia calcification. ${ }^{7}$

In general, brain calcinoses are more commonly found in patients aged 30 to 60 years, with the prevalence increasing with age. Findings also depend on the profile of the center where data are collected: most of them will be highly biased towards a given level of severity, gender, age range, or medical area of expertise, e.g., neurology, psychiatry, radiology, or geriatrics. Over a 1-year period, 4,219 consecutive CT scans were screened for basal ganglia calcification and 14 patients with calcification were identified. Calcifications on CT scan were bilateral in 12 cases and unilateral in two; the globus pallidus was the site of calcification in 13 of the 14 patients, and bilateral dentate nucleus calcification was seen in one patient. ${ }^{1}$ Eskandary et al. found three cases of abnormal calcification, in the pineal region, basal ganglia, and temporal horn area, amongst 3,000 CTs of head trauma patients in an emergency facility, at a mean age of $32 \pm 17.76 .^{8}$ Ostling et al. studied the cross-sectional relationship between psychotic symptoms and basal ganglia calcification in a population sample of nondemented subjects, all 85 years old: 86 were mentally healthy, 11 psychotic, 21 had mood disorders, and 20 had anxiety disorders. Basal ganglia calcifications on CT were observed in $19 \%$ of the mentally healthy and in $64 \%$ of nondemented individuals with hallucinations or delusions. No significant association was found between calcifications and mood or anxiety disorders, and the authors finally highlighted that basal ganglia calcification was strongly associated with

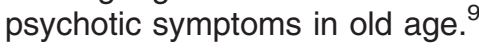

Hopefully, this body of knowledge will contribute to increase awareness concerning IBGC and, in the long run, also contribute to improve our understanding of other neuropsychiatric conditions that may mimic IBGC, with similar symptoms, such as dementia, parkinsonism, psychosis, and migraine.

\section{Disclosure}

The authors report no conflicts of interest.

\section{References}

1 Manyam BV. What is and what is not 'Fahr's disease'. Parkinsonism Relat Disord. 2005;11:73-80.

2 Oliveira JR, Steinberg SS. Far from rare: revisiting the relevance of idiopathic basal ganglia calcifications. Neurol Sci. 2010;31:679.

3 Oliveira JRM. Managing idiopathic basal ganglia calcification ("Fahr's disease"). New York: Nova Publishing; 2012.

4 Wang C, Li Y, Shi L, et al. Mutations in SLC20A2 link familial idiopathic basal ganglia calcification with phosphate homeostasis. Nat Genet. 2012;44:254-6.

5 Lemos RR, Oliveira MF, Oliveira JR. Reporting a new mutation the SLC20A2 gene in familial idiopathic basal ganglia calcification. Eur $\mathrm{J}$ Neurol. 2013;20:e43-4.

6 Nicolas G, Pottier C, Maltete D, Coutant S, Rovelet-Lecrux A, Legallic $S$, et al. Mutation of the PDGFRB gene as a cause of idiopathic basal ganglia calcification. Neurology. 2013;80:181-7.

7 McClellan RL, Eisenberg RL, Giyanani VL. Routine CT screening of psychiatry inpatients. Radiology. 1988;169:99-100.

8 Eskandary H, Sabba M, Khajehpour F, Eskandari M. Incidental findings in brain computed tomography scans of 3000 head trauma patients. Surg Neurol. 2005;63:550-3; discussion 553.

9 Ostling S, Andreasson LA, Skoog I. Basal ganglia calcification and psychotic symptoms in the very old. Int $\mathrm{J}$ Geriatr Psychiatry. 2003;18:983-7. 